\title{
Strong Support Effects on the Insulator to Metal Transition in Supported Pt Clusters as Observed by X-ray Absorption Spectroscopy
}

\author{
David E. Ramaker, ${ }^{* \dagger}$ Michiel K. Oudenhuijzen, ${ }^{*}$ and Diederick C. Koningsberger \\ Chemistry Department, George Washington University, Washington, D. C. 20052, and Department of Inorganic \\ Chemistry and Catalysis, Debye Institute, Utrecht University, P.O. Box 80083, \\ 3508 TB Utrecht, The Netherlands
}

Received: November 22, 2004

\begin{abstract}
The development of a new in situ probe of metallic character in supported metal clusters utilizing X-ray absorption spectroscopy is described. The technique is based on the extent of screening of the core-hole left in the neutral final state after the X-ray absorption. The technique allows for the clear differentiation between local interatomic charge transfer and more delocalized metallic screening. The particle size at the metalinsulator transition is found to depend strongly on the electron richness of the support oxygen atoms (i.e., ionic vs covalent oxides). Pt particles on supports with electron poor oxygen atoms (covalent) show metallic screening for sizes as small as $12 \AA$ in diameter. In contrast, on supports with electron rich oxygen atoms (ionic) the Pt particles do not show metallic behavior until around $20 \AA$. The wide variation of previously reported estimates of the particle size at which the insulator to metal transition occurs is explained, giving a consistent picture for the onset of metallic character, and the reasons for the strong support effect.
\end{abstract}

\section{Introduction}

There is great interest in deepening our understanding of metal atom clusters, particularly in the solid state. ${ }^{1}$ Such clusters are of fundamental interest because they span the range from very small molecular clusters having quantized states to relatively large microcrystalline clusters having quasi-continuous bands. They constitute new types of nanoscale materials with unique properties. The evolution of the electronic structure with cluster size is of particular interest; e.g., at what size, $d_{t}$, does the cluster become metallic? ${ }^{2}$ The subject of electronic or "quantum size effects" in metal particles has been previously reviewed. ${ }^{3}$

One of the problems in studies of the insulator-metal transition in these materials is finding some definitive experimental probes. Different experimental probes may measure different aspects of the electronic structure through the transition, causing different conclusions to be reached as to the onset of metallic character. ${ }^{4}$ A clear understanding of the transition, if successfully exploited, could pave the way for precise control of nanoscale properties and thus lead to a broader range of material applications, such as in novel catalysts, gas sensors, electronic materials, etc. If these applications are to be realized, however, the effect of the interaction of a nanoscale cluster with its supporting substrate or surroundings must also be quantified. ${ }^{5}$ Thus, the experimental probe must be applicable in situ under general conditions.

The range of probes for such metal-insulator transitions is very broad with each probe having its own strengths and weakness as summarized by Dowben: ${ }^{4}$

(a) Direct conductivity measurements are difficult to apply to free clusters, and even to two-dimensional systems.

(b) Spectroscopic measurements, such as electron energy loss spectroscopy (EELS), infrared (IR) and Raman spectroscopy,

* To whom correspondence should be addressed. Telephone: 202-9946934. Fax: 202-994-5873. E-mail: ramaker@gwu.edu.

George Washington University.

$\doteqdot$ Utrecht University. can probe the very small energy excitations across the Fermi level $\left(E_{\mathrm{f}}\right)$ or band gap by changes in the peak shape such as via the Drude tail in photoemission. However, such line shape changes can also be caused by a number of experimental effects making these measurements difficult.

(c) Measurements of the dielectric response from the optical properties and plasmon losses have similar problems.

(d) Work function and ionization potential measurements, although in widespread use for this purpose, may not be a good measure of the metallicity.

(e) Observation of the screening of the core-hole and the creation of excitons in core-level excitation has become a favored technique for observing the metal-insulator transition; the most utilized here has been core-level X-ray photoelectron spectroscopy (XPS). ${ }^{6}$

This paper describes the development of a new probe of metallic character utilizing core-level X-ray absorption spectroscopy (XAS), which has several important advantages over XPS:

(a) Because of its very nature (photons in and photons out using transmission or fluorescence yield mode), XAS can be applied in situ under general conditions, as opposed to XPS, which requires ultrahigh vacuum (UHV) conditions.

(b) Further, the XAS data provide simultaneously information about the cluster size from the extended X-ray absorption fine structure (EXAFS).

(c) Finally, the final state of the XAS excitation in the near edge (i.e., the Pt $5 d$ valence band) region does not change the charge on the cluster, so that the interaction with the support or surroundings is not different from the initial state. In contrast, XPS leaves a positive charge on the cluster, which requires a separation of the contributions arising from screening by the cluster itself and that arising from polarization by the support. ${ }^{7}$ This separation can be difficult.

The XAS technique developed here will separately reveal intra-atomic screening and metallic screening, a critical advan- 
tage of this technique over all previous experimental approaches. Although the technique is applied here under vacuum conditions, it can in principle be used under operando conditions. The results will show that the support properties (i.e., electron rich or poor oxygen atoms) can critically alter the metallicity of the cluster and that these effects exist already in the ground state. It should be understood that in ionic supports the oxygen atoms will be more electron rich than in covalent supports. When thinking of the supports as Lewis acids or bases in catalysis, the ionic oxides serve as a more basic support and the covalent oxides as a more acidic support. In the discussion section, the previous work on quantum size effects and the estimates of where the conduction onset occurs is summarized. These widely varying estimates are related and understood in the context of this work.

\section{Methods}

A. Sample Preparation and Data Collection. The newly developed technique is applied to Pt clusters supported on high surface area alumina or silica modified by doping with promoters such as $\mathrm{Cl}$ or $\mathrm{K}$ (denoted as $\mathrm{Cl}-\mathrm{Al}_{2} \mathrm{O}_{3}$ or $\mathrm{K}-\mathrm{SiO}_{2}$ ) to alter the electron richness of the support oxygen atoms. Data for $\mathrm{Pt}$ dispersed in zeolite LTL with various $\mathrm{K}^{+}$contents has also been utilized. ${ }^{8}$ It has been shown elsewhere that this cation exchange induces profound changes in the electron richness of the zeolitic support oxygens ${ }^{9}$ and, related to that, different catalytic properties of the supported clusters. ${ }^{9}$ The preparation of these samples has been described previously. ${ }^{8,10}$ The $\mathrm{Al}_{2} \mathrm{O}_{3}$ support (pseudobohemite, Criterion) was impregnated with a solution of $\mathrm{H}_{2-}$ $\mathrm{PtCl}_{6}(2$ wt \% Pt), dried, calcined at different temperatures, and further reduced at $623 \mathrm{~K}$. The silica support was prepared by ion-exchange of $\mathrm{SiO}_{2}$ microspheres (Davison grade 644, 284 $\mathrm{m}^{2} / \mathrm{g}, 1.12 \mathrm{~cm}^{3} / \mathrm{g}$ ) with an amount of $\mathrm{KOH}$ followed by calcination at $400{ }^{\circ} \mathrm{C}$. Pt was added by incipient wetness impregnation using an aqueous solution of $\mathrm{Pt}\left(\mathrm{NH}_{3}\right)_{4}\left(\mathrm{NO}_{3}\right)_{2}$ followed by calcination at $225^{\circ} \mathrm{C}$. The acidity of the LTL zeolite was varied by impregnating a commercial K-LTL zeolite with $\mathrm{KNO}_{3}$ or exchanging it with $\mathrm{NH}_{4} \mathrm{NO}_{3}$ to give different $\mathrm{K} / \mathrm{Al}$ ratios. Pt (1 wt \%) was added as described above followed by drying at $120{ }^{\circ} \mathrm{C}$.

The samples (typically $120-150 \mathrm{mg}$ ) were pressed into selfsupporting wafers and placed in an in situ stainless steel cell with controlled atmosphere. ${ }^{11}$ The samples were dried in flowing He for $1 \mathrm{~h}$ at $423 \mathrm{~K}$ and reduced for $1 \mathrm{~h}$ in $\mathrm{H}_{2}$ at $623 \mathrm{~K}$. Finally, the hydrogen was pumped off the Pt surface by evacuating for $1 \mathrm{~h}$ at $473 \mathrm{~K}$. After the evacuation, the samples were cooled to liquid nitrogen temperature, at which temperature the spectra were recorded. During the cooling and spectrum recording at liquid nitrogen temperatures, a vacuum better than $5 \times 10^{-3}$ $\mathrm{Pa}$ was maintained.

The XAS spectra of the Pt/LTL and Pt/K-SiO 2 samples were taken at the SRS laboratories (station 9.2) in Daresbury, U.K.; the spectra of the $\mathrm{Pt} / \mathrm{Cl}-\mathrm{Al}_{2} \mathrm{O}_{3}$ samples were taken at the ESRF (BM 29) in Grenoble, France, and at the HASYLAB (X1.1) in Hamburg, Germany. These measurements were done in transmission mode using ion chambers filled with Ar to have an X-ray absorbance of $20 \%$ in the first and $80 \%$ in the second ion chamber. The monochromator was detuned to $50 \%$ maximum intensity to avoid higher harmonics present in the beam.

B. Data Analysis and Model Development. The method described here to analyze the screening effects in the various Pt catalysts uses line shape and intensity changes in the X-ray absorption edges, not changes in the absolute energy of the absorption edge. Figure 1 shows as an example the $\mathrm{L}_{3}$ and the

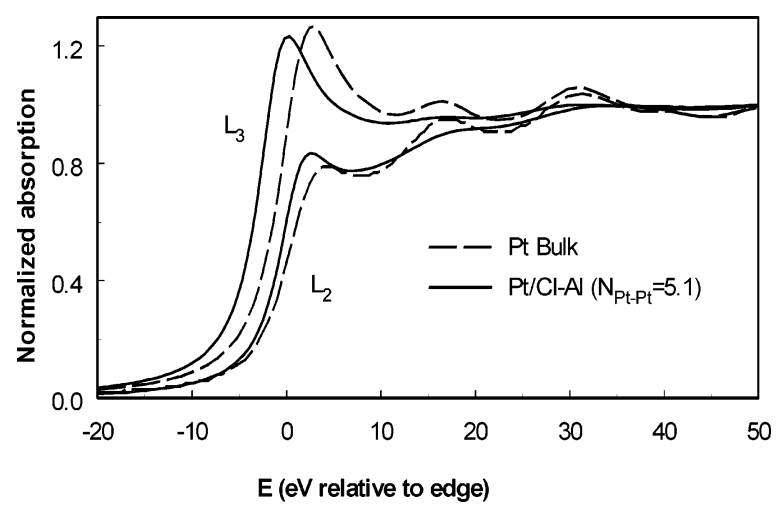

Figure 1. $\mathrm{Pt}_{3}$ and $\mathrm{L}_{2}$ edges (aligned as described in the text) for $\mathrm{Pt} / \mathrm{Cl}-\mathrm{Al}_{2} \mathrm{O}_{3}$ (solid line) $(N=5.1, \mathrm{Pt}$ particle size around $0.7 \mathrm{~nm})$ and for bulk Pt (dotted line).

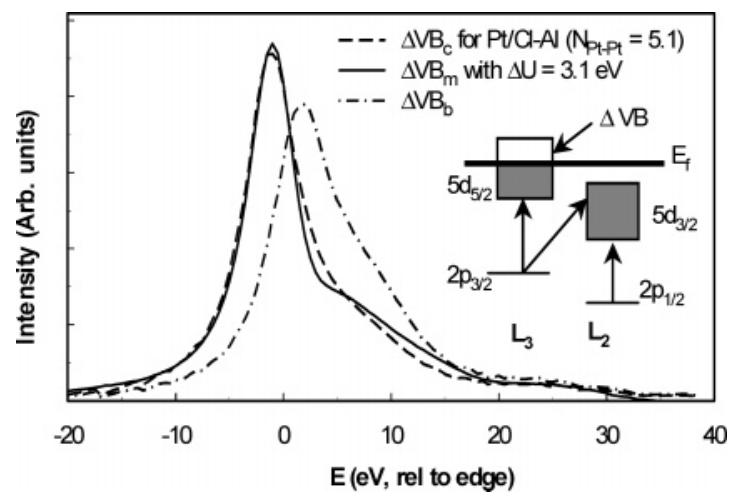

Figure 2. Comparison of $\left|\Delta V B_{\text {bulk }}\right|,\left|\Delta V B_{\text {cluster }}\right|$, and $\left|\Delta V B_{\text {model }}\right|$, where $\left|\Delta \mathrm{VB}_{\text {model }}\right|$ was obtained using eqs $1-3$ with an optimal $\Delta U$ value of $3.1 \mathrm{eV}$. Here $\left|\Delta \mathrm{VB}_{\text {bulk }}\right|$ and $\left|\Delta V \mathrm{~B}_{\text {cluster }}\right|$ have been area normalized. Insert: Illustration of the spin-orbital coupling effects in the X-ray absorption $\mathrm{L}_{2,3}$ edge spectra and $5 \mathrm{~d}$ valence band. The energy scale is nonlinear; i.e., those for the $2 \mathrm{p}$ and $5 \mathrm{~d}$ levels are very different.

$\mathrm{L}_{2} \mathrm{X}$-ray absorption edges of $\mathrm{Pt} / \mathrm{Cl}-\mathrm{Al}_{2} \mathrm{O}_{3}$ and $\mathrm{Pt}$ foil. The much smaller whiteline intensity near the edge in the $\mathrm{L}_{2}$ data compared to the $\mathrm{L}_{3}$ in transition metal samples is well-known and arises from spin-orbit interactions in the $\mathrm{Pt} 5 \mathrm{~d}$ state and the preferred selection rules as illustrated in the insert of Figure $2 .{ }^{12}$ The $5 \mathrm{~d}_{5 / 2}-5 \mathrm{~d}_{3 / 2}$ spin-orbital splitting is $\sim 1.5-2.5 \mathrm{eV}$ in $\mathrm{Pt}$, and the results reported here indicate this splitting depends on even the particle support. The $\mathrm{L}_{3}$ edge reflects the empty levels of both the $\mathrm{d}_{5 / 2}$ and $\mathrm{d}_{3 / 2}$ bands weighted as $\mathrm{d}_{5 / 2} / \mathrm{d}_{3 / 2} \approx 6 ;{ }^{12}$ however, the $L_{2}$ edge reflects only the $d_{3 / 2}$ level. The symbol $\Delta V B$ is utilized to indicate the difference in the empty valence band in the $\mathrm{L}_{3}$ minus that in the $\mathrm{L}_{2}$; i.e., $\Delta \mathrm{VB}=\mu\left(\mathrm{L}_{3}\right)-\mu\left(\mathrm{L}_{2}\right)$ where $\mu\left(\mathrm{L}_{n}\right)$ is the absorption cross-section at the $2 \mathrm{p}_{n / 2}$ edge. The difference, $\triangle \mathrm{VB}$, between the $\mathrm{L}_{3}$ and $\mathrm{L}_{2}$ data isolates the whiteline intensity and provides a spectral measure of the valence-band 5d empty density of states (DOS). However, this spectral measure of the empty DOS is modified by the presence of the core-hole consistent with the final-state rule. ${ }^{13}$

To align the Fermi levels in the $\mathrm{L}_{3}$ and $\mathrm{L}_{2}$ edges, the two spectra have to be placed on the same relative energy scale before the subtraction procedure can be carried out. For all samples, the energy at 0.6 the intensity of the step height of the $\mathrm{L}_{2}$ edge is set to a reference energy of $0 \mathrm{eV}$. At higher energy, the EXAFS oscillations are influenced only by the geometry around the X-ray absorber atom, not by final state effects. Therefore, the $\mathrm{L}_{3}$ EXAFS oscillations of the Pt catalysts are aligned carefully with the comparable EXAFS oscillations 
of the accompanying $\mathrm{L}_{2}$ spectra. The $\mathrm{L}_{3}$ and $\mathrm{L}_{2}$ edges as presented in Figure 1 are aligned on the nodes of the EXAFS oscillations.

(a) Model Development for the $\Delta V B$ Spectral Line Shape. A model needs to be developed to analyze the spectral line shape of $\triangle \mathrm{VB}$ and thereby gain information about the core-hole electron attraction as a function of particle size and support. Figure 2 compares the $\Delta \mathrm{VB}$ obtained for bulk $\mathrm{Pt}$ with that for a Pt cluster supported on alumina with coordination number $N$ = 5 (approximately $7 \AA$ diameter) as obtained by analysis of the EXAFS data. The difference in line shape here reveals that the core-hole electron attraction $U$ in the cluster is different than it is in the bulk. Because of metallic screening, $U$ is near zero in bulk Pt. A reasonable average measure of this $U$ in the cluster can be obtained by modifying the bulk line shape, $\Delta \mathrm{VB}_{\mathrm{b}}$, by utilizing the Anderson $\mathrm{Z}+1$ impurity model ${ }^{14,15}$

$$
\left|\Delta \mathrm{VB}_{\text {model }}(E)\right|=\frac{\left|\Delta \mathrm{VB}_{\text {bulk }}(E)\right|}{[1-\Delta U I(E)]^{2}+\left[\pi \Delta U\left|\Delta \mathrm{VB}_{\text {bulk }}(E)\right|\right]^{2}}
$$

where $\left|\Delta \mathrm{VB}_{\text {model }}(E)\right|$ is the normalized model $\Delta \mathrm{VB}$ at energy $E ;\left|\Delta \mathrm{VB}_{\text {bulk }}(E)\right|$ is the normalized bulk $\Delta \mathrm{VB}$, obtained from a Pt foil; $\Delta U$ is the core-hole-electron attraction in the cluster, relative to that in the bulk ( $U \approx 0)$, where $\Delta U$ is chosen such that $\Delta \mathrm{VB}_{\text {model }}$ is closest to the $\Delta \mathrm{VB}$ of the measured cluster, $\Delta \mathrm{VB}_{\text {cluster; }}$; and $I(E)$ is the Hilbert transform at energy $E$ :

$$
I(E)=\int_{\epsilon=-\infty}^{\epsilon=+\infty} \frac{\left|\Delta \mathrm{VB}_{\text {bulk }}(\epsilon)\right|}{E-\epsilon} \mathrm{d} \epsilon
$$

The parallel bars indicate normalization, given by

$$
|\Delta \mathrm{VB}(E)|=\Delta \mathrm{VB}(E) / A(\Delta \mathrm{VB})
$$

and

$$
A(\Delta \mathrm{VB})=\int_{\epsilon=-\infty}^{\epsilon=+\infty} \Delta \mathrm{VB}(\epsilon) \mathrm{d} \epsilon
$$

In practice the integrals are calculated from -40 to $+40 \mathrm{eV}$ around the absorption edge, since the integrands are negligible beyond these limits.

(b) Model Development for the $\Delta V B$ Intensity (Area Ratio, $R$ ). Information about the number of holes and the type of screening mechanism as a function of metal particle size and support can be obtained from the ratio of the integrated areas, $R(N)=A\left(\Delta \mathrm{VB}_{\text {cluster }}\right) / A\left(\Delta \mathrm{VB}_{\text {bulk }}\right)$, where it is made explicit that $R$ depends on the cluster size (i.e., $N$ ). First the different possible screening mechanisms are discussed, and then a model will be developed for $R$ as a function of the number of holes without and with core-hole screening.

The Different Dynamic Screening Mechanisms. A core-hole is dynamically screened by a series of processes as summarized in Figure 3. This dynamic screening, as discussed extensively by Mahan, ${ }^{16}$ Gadzuk, ${ }^{17}$ von Barth and Grossman, ${ }^{13}$ and others, ${ }^{18,19,20}$ depends on the time scale over which the effective potential was changed. The various processes are schematically shown in Figure 3. For slow, nearly adiabatic changes (such as that examined here near threshold), the entire system is in the ground state of the potential determined by the instantaneous charge density. Thus initially $\left(10^{-16} \mathrm{~s}\right)$, the relaxation occurs by interatomic charge transfer (ICT) from neighboring atoms to the atom with the core-hole. Some time later $\left(10^{-14} \mathrm{~s}\right)$, the screening is accomplished by $5 \mathrm{~d}$ electron-hole pair excitations at $E_{\mathrm{f}}$, which facilitates the nonlocal electron hopping or metallic

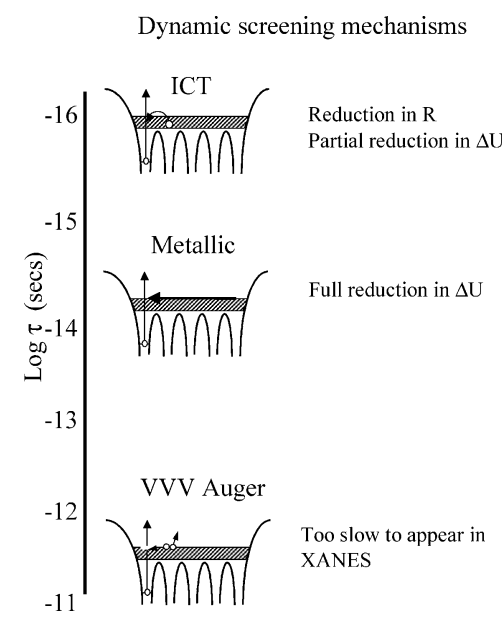

Figure 3. A schematic illustration of the various screening processes and the time scale on which they occur.

screening. Such metallic screening is generally much more efficient in a metal, so if the latter occurs, the initial ICT screening is reversed, and the effective core-hole electron attraction is reduced to zero by the metallic screening. In the absence of either of these relaxation mechanisms (e.g., in a small cluster or molecule), valence-valence-valence (VVV) Auger processes may allow for rearrangement of charge in the cluster MOs. ${ }^{17}$ However, such VVV Auger processes are even slower $\left(10^{-11} \mathrm{~s}\right)$ than the CVV Auger process $\left(10^{-13} \mathrm{~s}\right)$ that determines the lifetime of the $\mathrm{Pt} \mathrm{L}_{3}$ core-hole. Thus, in this sense the XAS does not reflect the completely adiabatic case, since the final VVV processes do not get a chance to occur in the absence of ICT and metallic screening.

Both the ICT and metallic screening mechanisms depend critically on the DOS near $E_{\mathrm{f}}$. Frölich ${ }^{21}$ and $\mathrm{Kubo}^{22}$ pointed out long ago that a cluster may exhibit metallic behavior when the average spacing between the electronic levels becomes smaller than $k T$ ( $k$ is Boltzman's constant) and the discrete energy levels begin to form a quasi-continuous band. Formulated in terms of the DOS, the transition to metallic behavior occurs when the DOS within $0-0.1 \mathrm{eV}$ of $E_{\mathrm{f}}$ exceeds $1 / k T$ (or approximately 40 states/(eV-cluster $)) .{ }^{23}$ In contrast, the ICT process depends on the DOS within $0-3 \mathrm{eV}$ of $E_{\mathrm{f}}$. In wideband materials, ICT screening sets in not only at shorter times but in clusters smaller than $d_{\mathrm{t}}$, and as the cluster size increases above $d_{\mathrm{t}}$, ICT screening is reversed and $\Delta U$ sharply decreases. However, for very small particles or $\mathrm{Pt}$ atoms with low coordination near edges or corners, the bandwidth $(\Gamma)$ may be sufficiently narrow that electron correlation effects characterized by an electron-electron interaction $U$ (i.e., $\mathrm{VV}$ interaction to be distinguished from the core-hole electron interaction $\Delta U$ discussed above) may be important. In this case, the Mott-Hubbard ${ }^{24,25}$ criterion for electron conduction, $U<\Gamma$, must also be satisfied for metallic character to set in. This will be an important point to be discussed further below.

Model for Variation in $R=h_{5 / 2}-h_{3 / 2}$ Assuming No ICT Screening. The variation in $R(N)$ with $N$ is complex, reflecting in part the variation in the average number of holes/atom, $\Delta h(N)$ $=h_{5 / 2}-h_{3 / 2}$ in the $\mathrm{d}$ band with $N$, and in part the effects of ICT screening. These two contributions need to be separated, and this can be done by developing a simple model for $\Delta h(N)$.

The total number of holes/Pt atom in the cluster increases with cluster size, i.e., with $N$. Assuming Goddard' ${ }^{26}$ generalized valence bond (GVB) model for bonding in transition metals, each Pt atom in the bulk participates in two interstitial bond orbitals (IBOs). This occurs because half of the tetrahedrons in 


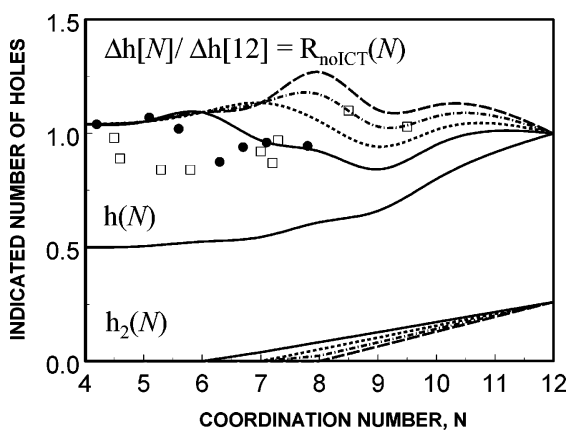

Figure 4. Experimental $R(N)$ (area ratio) points for covalent (solid circles) and ionic supports (open squares) compared with four different estimates of $R_{\text {noICT }}(N)$, eq 4, assuming four different estimates of $N_{\text {th }}$, the value of $N$ where the $\mathrm{L}_{2}$ band opens (the assumed value of $N_{\text {th }}$ for each estimate can be obtained by reading the point where the corresponding $h_{2}(N)$ line, the number of $\mathrm{L}_{2}$ holes/Pt atoms, goes to zero on the $N$ scale). Also plotted is the total number of holes, $h(N)$, versus $N$ (solid line) which is equal to $1-0.5 D(N)$ where $D$ is the dispersion (see Figure 7 below and the text for an explanation). The optimum value of $N_{\text {th }}$ is shown to be around 6 for the covalent and 7.5 for the ionic supports.

a bulk metal contain an IBO, and each Pt atom participates in 4 tetrahedrons $(N=12$ in bulk $\mathrm{Pt})$, i.e., contributes $1 / 2$ electron to each IBO. Therefore each bulk $\mathrm{Pt}$ atom contributes one $\mathrm{sp}$ electron total, and the remaining electrons go into the d band, giving a $\mathrm{sd}^{9}$ electron configuration in the bulk. At a $\operatorname{Pt}(111)$ surface, half of the IBOs are broken, so each surface Pt atom contributes to one IBO and just $1 / 2$ of an electron to the $\mathrm{sp}$ bonding, giving a $\mathrm{s}^{0.5} \mathrm{~d}^{9.5}$ electron configuration. Thus the number of holes in the $\mathrm{d}$ band goes from 1 in the bulk to $1 / 2$ at the surface. Although flat faces on a cluster become smaller and the number of edge/corner atoms increases as the cluster becomes still smaller, the GVB calculations indicate that the electron configuration does not deviate significantly from $\mathrm{s}^{0.5} \mathrm{~d}^{9.5}$ as the cluster becomes very small (i.e., each Pt still contributes to at least one IBO). The fraction of surface atoms at each $N$ is known from the dispersion, $D(N)$; therefore the total number of holes/atom, $h[N]=1-0.5 D(N)$ at $N$, can easily be obtained as plotted in Figure 4.

The distribution of these holes between the $\mathrm{L}_{3}$ and $\mathrm{L}_{2}$ bands is a little more difficult to obtain, because the spin-orbit splitting is not easy to estimate. Nevertheless, it is clear that the bandwidth increases with the Pt coordination, $N$, so that the number of holes/atom in the $\mathrm{L}_{2}$ band, $h_{2}[N]$, must increase with $N$. Therefore, a reasonable approximation is $h_{2}\left[\mathrm{~N}>N_{\text {th }}\right]=$ $h_{2}[12]\left(N-N_{\text {th }}\right) /\left(12-N_{\text {th }}\right)$, where $N_{\text {th }}$ is the threshold $N$ or onset for emptying of the $\mathrm{L}_{2}$ band as the cluster size increases, and $h_{2}[12]$ is the number of holes in the $\mathrm{L}_{2}$ band in bulk Pt. It is then assumed that the remainder of the holes goes into the $\mathrm{L}_{3}$ band or $h_{3}[N]=h[N]-h_{2}[N]$. Therefore the area ratio, $R_{\text {noICT }}(N)$, assuming no ICT screening, can be defined more specifically in terms of the $h$ 's by the following equation:

$$
\begin{aligned}
& R_{\mathrm{noICT}}(N)=\left(h_{3}[N]-h_{2}[N]\right) /\left(h_{3}[12]-h_{2}[12]\right)= \\
& \left(h[N]-2 h_{2}[N]\right) /\left(h[12]-2 h_{2}[12]\right)=(1 .-0.5 D(N)- \\
& \left.2 h_{2}[12]\left(N-N_{\mathrm{th}}\right) /\left(12-N_{\mathrm{th}}\right)\right) /\left(1.0-2 h_{2}[12]\right)
\end{aligned}
$$

where $N-N_{\text {th }}$ is equal to zero for $N$ less than $N_{\text {th }}$.

Reduction in $R$ Due to ICT Screening. Large deviations between the experimental $R(N)$ values and $R_{\text {noICT }}(N)$ can occur when ICT screening is operative. FEFF8 calculations were performed to investigate the influence of ICT screening on the ratio of the integrated areas. Figure 5 shows DOS from self-

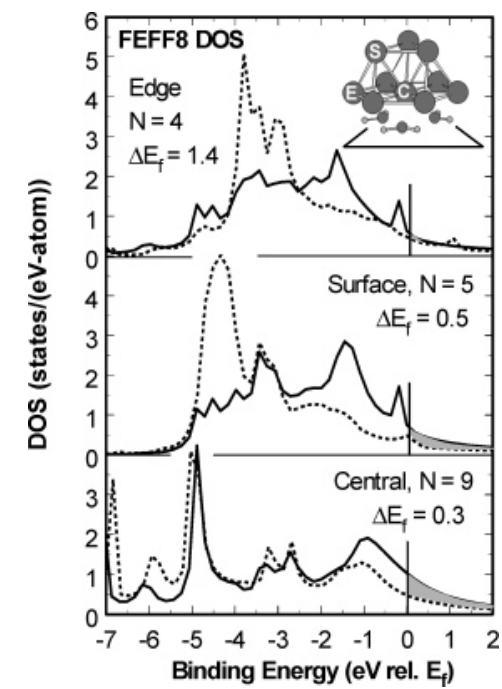

Figure 5. Pt d DOS obtained from a FEFF8 calculation for a $\mathrm{Pt}_{10^{-}}$ $(3,7)$ cluster on a support mimicked by three water molecules as shown. The DOS are shown for the edge (E), surface (S), and center (C) atoms without (solid line) and with (dotted line) the core-hole. The DOS obtained with the core-hole have been shifted by the amounts $\left(\Delta E_{\mathrm{f}}\right)$ indicated for each case to obtain agreement with the no-hole results, reflecting the magnitude of the shift of the Fermi level on the absorber atom due to the presence of the core-hole. The shaded areas indicate the screening charge. The coordination number $N$ at each site is also given.

consistent field, full multiple scattering calculations as implemented in the FEFF8 code $^{27}$ on the supported $\mathrm{Pt}_{10}$ cluster illustrated. The support is mimicked by the 3 water molecules as shown; the $\mathrm{O}$ atoms represent the $\mathrm{O}$ atoms in the support, and the $\mathrm{H}$ atoms effectively terminate the cluster and represent the $\mathrm{Si}$ or $\mathrm{Al}$ atoms such as that in a zeolite. The DOS are shown from calculations with and without a core-hole on the 3 different site symmetries of the cluster (edge, surface, and central). The calculation without a core-hole mimics the case when metallic screening reduces the core-hole electron attraction by a more delocalized electron accumulation and polarization around the core-hole atom.

Notice first that the magnitude of the unoccupied DOS increases significantly with the coordination number $(4,5$, or 9) of the Pt absorber site. The DOS for each core-hole site has been shifted by the energy indicated $\left(\Delta E_{\mathrm{F}}\right.$ in Figure 5$)$ to give best alignment with the no-core-hole calculation. This energy shift represents the increase in binding energy of the Pt $5 \mathrm{~d}$ band on the absorber atom, or the extent to which the core-hole remains unscreened. Notice also the sharp reduction in this shift with the increase in the magnitude of the ICT screening charge as indicated by the shaded areas.

Figure 6 shows the calculated $\Delta \mathrm{VB}$ at the 3 different sites on the $\mathrm{Pt}_{10}$ cluster, obtained by taking the difference $\mu\left(\mathrm{L}_{3}\right)-$ $\mu\left(\mathrm{L}_{2}\right)$ from FEFF8 exactly as $\triangle \mathrm{VB}$ is obtained experimentally. Thus these theoretical results should directly mimic the experimental results and show the variation in $R(N)$ with $N$. The calculated $\triangle \mathrm{VB}$ values for the 3 different cluster sites are shown, assuming metallic screening (no core-hole) and with ICT screening (with core-hole). In each instance $E_{\mathrm{f}}$ was shifted downward by the amounts indicated by $\Delta E_{\mathrm{F}}$ in Figure 5 , since in the absence of the VVV decay process, the "effective" $E_{\mathrm{f}}$ is indeed shifted downward on the absorber atom by these amounts.

The increase of the $\triangle \mathrm{VB}$ intensity with $N$ assuming full metallic screening (no core-hole, light solid line in Figure 6) reflects the increase in empty DOS with $N$ shown in Figure 5. 


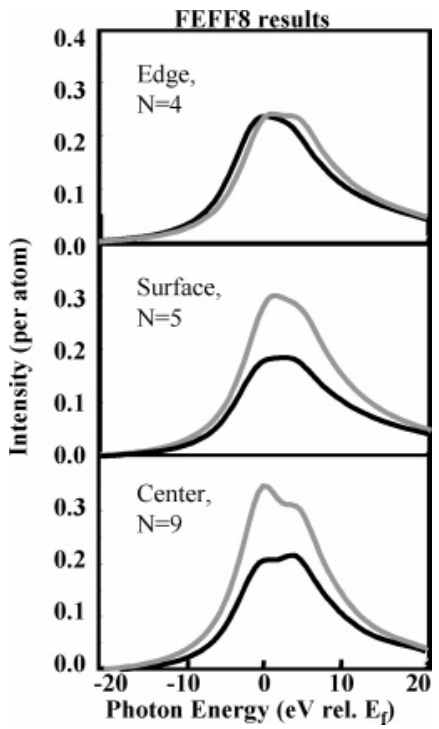

Figure 6. The FEFF8 calculated $\triangle \mathrm{VB}$ for the three sites on the $\mathrm{Pt}_{10}$ cluster indicated in Figure 5, obtained with (dark lines) and without (light lines) the core-hole.

However, the ICT-screened $\triangle \mathrm{VB}$ (with core-hole, dark solid line) does not show this increase; indeed now $\triangle \mathrm{VB}$ is largest for the edge $(N=4)$ sites. The reduction in $\Delta \mathrm{VB}$ intensity upon introduction of the core-hole occurs because the ICT significantly reduces the unoccupied DOS on the absorber atom. This reduction is much greater for the larger coordination sites, obviously because the ICT is much greater. When the ICT is nearly negligible, the unscreened $\Delta \mathrm{VB}$ is actually slightly larger than the screened case. Also, the theoretical $\Delta \mathrm{VB}$ shifts to lower energy with decreasing $N$, reflecting an increased $U$.

As summarized in Figure 3, ICT screening reduces the $R(N)$ ratio on the atom with the core-hole, i.e., the $R(N)$ ratio obtained experimentally, from the $R_{\text {noICT }}(N)$ value that simply reflects the average $\Delta h(N) / \Delta h(12)$ ratio for all Pt atoms.

\section{Results}

Figure 7 plots the changes in the experimental $\Delta U(N)$ and $R(N)$ with cluster size (i.e., $N$ ) for ionic and covalent oxidic supports. It can be seen that $\Delta U(N)$ and $R(N)$ vary dramatically with cluster size and support properties. Note that $\Delta U(N)$ goes to zero and the ratio of the integrated areas, $R(N)=A\left(\Delta \mathrm{VB}_{\text {cluster }}\right) /$ $A\left(\Delta \mathrm{VB}_{\text {bulk }}\right)$, approaches 1 for the large clusters as expected, since the electronic structure in the larger clusters must eventually resemble that in the bulk.

The cluster size is determined from the experimental $\mathrm{Pt}-\mathrm{Pt}$ coordination number $N_{\mathrm{PtPt}}$ obtained from the EXAFS. Model calculations assuming spherical clusters with face-centered cubic (fcc) packing ${ }^{28,29}$ then allow us to give the approximate particle diameters, the total number of atoms $Z_{\text {total }}$, and dispersion $\left(Z_{\text {surface }} / Z_{\text {total }}\right)$. Note the very nonlinear scale in diameter on this linear coordination number axis. These scales show that for particles with $N$ around 5, the dispersion is near 1 , meaning that most of the atoms are surface atoms.

Figure 7 shows that the area ratio, $R(N)$, is greater than 1 at $N=4$ exactly as indicated by FEFF8 calculations as given above. This ratio can be used to determine $h_{2}[12] / h_{3}[12]$. The magnitude of the ratio $h_{2}[12] / h_{3}[12]$ has been controversial even for bulk Pt, with early estimates varying from 0.07 up to $0.34 .{ }^{12}$ The most reliable estimate from bulk band structure calculations at 0.34 appears to agree best with experimental data. ${ }^{12}$ An estimate can be obtained easily from the experimental data in

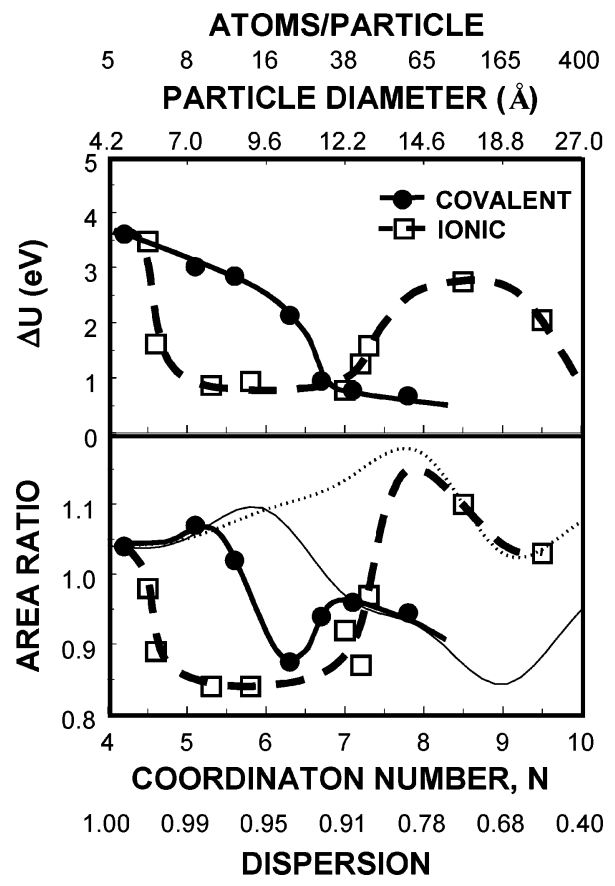

Figure 7. Comparison of the experimental $\Delta U$ and the area ratio $R=$ $A\left(\Delta \mathrm{VB}_{\mathrm{c}}\right) / A\left(\Delta \mathrm{VB}_{\mathrm{b}}\right)$ as a function of the $\mathrm{Pt}-\mathrm{Pt}$ coordination number $N$ for covalent or acidic (solid lines) and ionic or basic (heavy dashed lines) supports. The $N$ values were obtained from EXAFS analyses summarized elsewhere. ${ }^{10,11,17,18}$ Also plotted are the optimum $R_{\text {nolCT- }}$ $(N)$ (lighted dashed line) results obtained from eq 4. The horizontal axis shows the estimated dispersion, cluster diameter, and number of atoms/particle as estimated assuming spherical clusters and fcc packing (see the text). Data shown include those for covalent (acidic) supports (i.e., for $\mathrm{LTL}[\mathrm{K} / \mathrm{Al}=0.63]$ zeolite and $\mathrm{Cl}-\mathrm{Al}_{2} \mathrm{O}_{3}, \mathrm{SiO}_{2}$ ) and for ionic (basic) supports $\left(\mathrm{LTL}[\mathrm{K} / \mathrm{Al}=1.25]\right.$ zeolite and $\mathrm{K}-\mathrm{Al}_{2} \mathrm{O}_{3}, \mathrm{~K}-\mathrm{SiO}_{2}$ ). The uncertainty in $N, U$, and $R$ is estimated as follows: $\delta N= \pm 0.2, \delta U=$ \pm 0.3 , and $\delta R= \pm 0.1$.

Figure 7, since at low $N$, ICT screening is negligible and $D(N)$ $=1$. It can be seen in Figure 7 that $R_{\text {noICT }}(N)$ is about 1.04 for $N=4$, regardless of support acidity. Using eq 4 and assuming $N=4$ is less than $N_{\text {th }}$ gives $R_{\text {noICT }}\left(N<N_{\text {th }}\right)$ equal to $0.5 /(1-$ $\left.2 h_{2}[12]\right)$ leading to $h_{2}[12]=0.26$. Since we estimate $h[12]=$ 1 hole/Pt atom, this gives $h_{2}[12] / h_{3}[12]=0.26 / 0.74=0.35$ in excellent agreement with theoretical calculations. This agreement helps to validate our model.

$R_{\text {noICT }}\left(N>N_{\text {th }}\right)$ can now be calculated as a function of $N$ using eq 4 with $h_{2}$ [12] fixed and with $N_{\text {th }}$ as a variable. The experimental results from Figure 7 , and also plotted in Figure 4 , can be used to determine $N_{\text {th }}$. The different lines in Figure 4 representing $R_{\text {noICT }}(N)$ and $h_{2}\left[N>N_{\text {th }}\right]=h_{2}[12]\left(N-N_{\text {th }}\right) /(12-$ $\left.N_{\text {th }}\right)$ were calculated using different values of $N_{\text {th }}$. The lines calculated with values of $N_{\text {th }}=6$ and 7.2 are found to provide the best agreement with the experimental data for the covalent and ionic supports, respectively, where it is assumed that the last two data points in each case are in regions where ICT screening is negligible. The experimental $R$ values at lower $N$ are significantly lower than the calculated values because of ICT screening as indicated above.

The calculated values for $R_{\text {noICT }}(N)$ are also included in Figure 7 as a dotted line for the supports with electron poor (covalent or acidic) oxygen atoms and a light solid line for the supports with electron rich (ionic or basic) oxygen atoms. In light of our model above, the experimental drop in $R(N)$ from the $R_{\text {noICT }}$ $(N)$ values occurs because the X-ray absorption near edge spectroscopy (XANES) reflects in part the local unoccupied DOS on the absorber atom in the final state, and this is reduced by ICT screening. Thus Figure 7 shows that significant ICT 
screening occurs between $N=5.5$ and 6.5 on supports with electron poor (acidic) oxygen atoms and between $N=4.5$ and 7.5 on supports with electron rich (basic) oxygen atoms.

The final decline of $\Delta U$ to near zero occurs at $N=6.5\left(d_{\mathrm{t}}=\right.$ $10 \AA$ ) for supports with electron poor oxygen atoms and way out at $N=9.5\left(d_{\mathrm{t}}=25 \AA\right)$ for supports with electron rich oxygen atoms. Thus the insulator to metal transition is very dependent on the electron richness of the support oxygen atoms.

\section{Discussion}

A. Validity of the Analysis Method. Figure 2 shows excellent agreement between the Anderson $\mathrm{Z}+1$ model $^{14,15}$ $\left(\left|\Delta \mathrm{VB}_{\text {model }}\right|\right.$ from eq 1$)$ and that for the cluster $\left|\Delta \mathrm{VB}_{\text {cluster }}\right|$ utilizing a $\Delta U$ of $3.1 \mathrm{eV}$. Note that the small shoulder in the experimental $\left|\Delta \mathrm{VB}_{\text {cluster }}\right|$ is nicely reproduced by the model. Note again that $\left|\Delta \mathrm{VB}_{\text {bulk }}\right|$ and $\left|\Delta \mathrm{VB}_{\text {cluster }}\right|$ are area normalized here, and eq 1 preserves this normalization. The $\mathrm{Z}+1$ impurity model chosen here assumes that the impurity states ( $5 \mathrm{~d}$ orbitals on atom with core-hole) are largely decoupled from the other host states, in contrast to the Clogston-Wolf model, which assumes that the impurity-host hybridization matrix element is unchanged from the host-host. ${ }^{30}$ The former is used for two reasons: it is sufficiently valid since we are interested only in the relative changes in $\Delta U$ with respect to bulk $\mathrm{Pt}$, and it is more convenient.

The size of $\Delta U(\approx 3.5 \mathrm{eV})$ for the very small clusters is consistent with previous estimates of $U(5 \mathrm{~d} 5 \mathrm{~d}) \approx 3-6 \mathrm{eV}$ obtained from CVV (core 5d 5d) Auger spectra for the nearly filled $5 \mathrm{~d}$ band transition metals when efficient metallic screening is shut off. ${ }^{31}$

Most interesting is the large difference in the behavior of the core-hole electron attraction for Pt particles on supports with electron poor vs electron rich oxygen atoms. This difference dramatically reveals the importance of the support in determining the nature and onset of the metal-insulator transition. It provides direct experimental evidence for a metal-support interaction.

B. Support Effect on Pt Hole Distribution and Screening. (a) Support Effect on Pt Particle Work Function. The very important differences in the $\Delta U(N)$ and $R(N)$ behavior between supports with covalent vs ionic support oxygen atoms can be understood using previously reported models for the support effect. As the covalency of the support increases, the electron richness of the support $\mathrm{O}$ atoms decreases. ${ }^{8,32}$ This decreased $\mathrm{O}$ electron richness changes the Madelung potential which shifts the Pt $5 d$ orbitals to higher binding energy, causing a higher work function. Feibelmann and Hamann ${ }^{33}$ first suggested such a change in the work function from theoretical calculations. This shift in the $5 \mathrm{~d}$ band was confirmed experimentally utilizing atomic XAFS and further theoretical calculations. ${ }^{8,10,34}$ This can easily be modeled with the $\mathrm{Pt}_{10}$ cluster illustrated in Figure 5, by changing the electron richness of the support oxygen atoms by small levels, say $0.05 \mathrm{e}^{-} / \mathrm{O}$ atom. Such a calculation (results not given here) shows the $5 \mathrm{~d}$ DOS shifts by nearly $1 \mathrm{eV}$, but the shift is not uniform. The central atom DOS on the cluster in Figure 5 moves to higher binding energy while the edge atom DOS moves much less on supports with electron poor oxygen atoms, since only the more interior atoms experience the full increased work function. The effect will be in the opposite direction on supports with electron rich oxygen atoms.

Thus electrons will move around the surface of the cluster with changing support covalency, and the holes will move in the opposite direction. The calculation mentioned above on the small $\mathrm{Pt}_{10}$ cluster reflects rearrangement of electrons from the

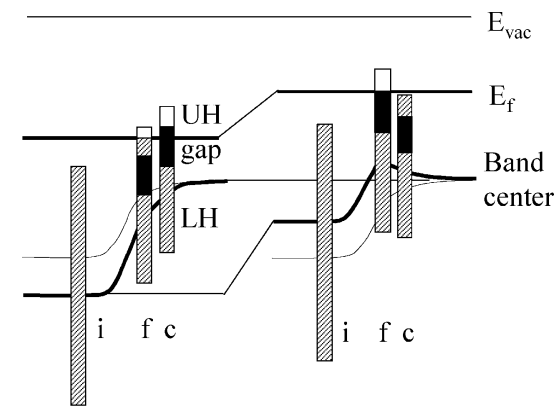

Covalent Ionic

Figure 8. Schematic showing the expected shift of the interior (i), face (f), and corner (c) $5 \mathrm{~d}$ valence bands on covalent (left) and ionic (right) supports relative to the vacuum level $\left(E_{\mathrm{vac}}\right)$ for clusters in the range $N=6-8$. The expected band center of these 3 bands on neutral supports is indicated by the light solid lines, and that for the covalent or ionic by the heavy lines. Within each band, the occupied (gray), band gap (solid black), and unoccupied (white) regions are indicated. The upper (UH) and lower (LH) Hubbard bands are labeled for one case, but they exist for both the corner and face bands on both supports.

edge to the central atom, suggesting that one can generalize the phenomenon and indicate that electrons move from lower to higher coordinated $\mathrm{Pt}$ atoms on supports with electron poor oxygen atoms, and the holes in the opposite direction, from higher to lower coordination. This redistribution of electrons or holes will have the effect of decreasing the net DOS at $E_{\mathrm{f}}$ on small- to medium-sized Pt clusters on supports with electron poor oxygen atoms. Tong et al. have experimentally verified this using NMR studies that show the local DOS at $E_{\mathrm{f}}$ indeed changes by the degree and nature of the ion exchange in the support $^{35}$ (i.e., electron richness of the support oxygen atoms) and confirms the direction of the charge rearrangement is exactly as indicated above.

(b) Effect of Work Function on Hole Distribution and Screening. Figure 8 schematically illustrates the effect of the support-induced changes in the work function on the hole distribution in supported clusters with $N$ in the range $7-8$. This figure shows decreasing band with and decreasing work function with decreasing coordination number (interior $>$ face $>$ corners). Here, we use interior, face, and corner to identify particular sites on an assumed larger cubo-octahedral cluster consisting of many more atoms than the 10 atom cluster illustrated in Figure 5 (these different sites will be further clarified below). For covalent supports, the work function is increased in the same order and decreased in the same order for ionic supports. The interior atoms have no holes on either support for these size clusters as illustrated, and this is confirmed by theoretical calculations. ${ }^{36}$ Thus although more holes exist in the cluster because of the larger IBO coordination of interior atoms, the holes do not necessarily remain on the interior atoms.

The experimental $\triangle \mathrm{VB}$ intensity derives from the average $R$,

$$
R(N)=\left[f_{\mathrm{c}}(N) R_{\mathrm{c}}+f_{\mathrm{f}}(N) R_{\mathrm{f}}+f_{\mathrm{i}}(N) R_{\mathrm{i}}\right]
$$

where the terms in brackets represent the fraction of each type of site, $f_{k}$ (significantly varying with $N$ ), and the area ratio $R_{k}$, at each type of site, $k\left(R_{k}\right.$ less variant on $\left.N\right)$. Figure 9 shows the fraction, $f_{k}(N)$, as determined from cluster calculations assuming a cubo-octahedral cluster. ${ }^{37}$ Gordon et al ${ }^{37}$ report four different site categories: corner, edge, face, and interior sites with $N$. Here we combine the edge and face site numbers together and give only three site categories in Figure 9; i.e., $k$ $=$ corner, face, and interior. Figure 9 shows also the number of 


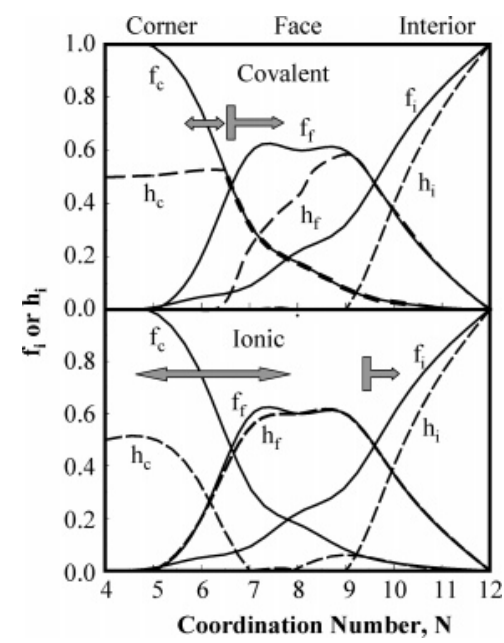

Figure 9. The fraction, $f$, of corner, face, and interior sites (solid lines) in cubo-octahedral clusters as a function of $N$, the coordination number, from ref 37. Also shown is the hole density, $h$, on each of the sites (dashed lines). When $h=f$, the hole occupancy, $n$, is 1 . The case where the holes fill in the order corner $<$ face $<$ interior is indicated at the top for the covalent supports, and that for the order face $<$ corner $<$ interior is at the bottom for ionic supports. The doubly sided horizontal arrow indicates the region where ICT screening is expected to be important in each case, and the blocked arrow pointing to the right indicates where metallic screening begins to set in.

holes per atom on each type of site, $h_{k}(N)$, such that $\sum_{k} h_{k}(N)=$ $h(N)$, and $h_{k}(N)=n_{k}(N) f_{k}(N)$. Here $n_{k}(N)$ is the occupation number varying from 0 to 1 with change in $N$.

Based on our model for the effects of the support (see Figure 8), on covalent supports the corner sites fill with holes first with increasing $N$, followed by the face, and then the interior. In contrast, for ionic supports the face sites fill first, and then the edge, followed by the interior. Further, for reasons to be stated below, it is assumed that each site can accept a maximum of one hole (i.e., maximum $n_{k}=1$ ). One hole exists on each atom when $h_{k}(N)=f_{k}(N)$ in Figure 9, otherwise less.

Figure 9 now shows that ICT screening (indicated by the shaded double arrow) occurs primarily at the corner sites on covalent supports and shuts off when $h_{\mathrm{c}}(N)$ begins to reach $f_{\mathrm{c}}$ $(N)$ (i.e., when $n_{\mathrm{c}}(N)$ approaches 1$)$. In contrast, on ionic supports ICT screening occurs on both corner and face sites and shuts off when $n_{\mathrm{f}}(N)$ begins to reach $f_{\mathrm{f}}(N)$ (i.e., when $n_{\mathrm{f}}(N)$ approaches $1)$. Figure 9 also shows that $n_{\mathrm{c}}(N)\left[n_{\mathrm{f}}(N)\right]$ approaches 1 around $N=6.5$ (7.5) on the supports with electron poor (rich) oxygens, just where the ICT screening decreases dramatically according to Figure 7. Further, metallic screening sets in when the number of holes on the face (interior) sites becomes significant $(>0.25)$ on the covalent (ionic) supports at $N=7$ (9.5), just where the experimental data in Figure 7 suggest that the transition occurs. This condition confirms that metallic screening is possible when the holes become evenly distributed among the "operational" sites (edge/face on acidic supports and face/edge on basic supports), and the DOS becomes greater than 40 states/eV-atom.

The agreement between the "transition" points in Figure 9 and the experimental data in Figure 7 suggests that the holes are strongly correlated on the edge and face sites, since the transition points in Figure 9 are based in part on the hole occupancy maximizing at 1 . Hole-hole repulsion only allows one hole per site. Strong repulsion or correlation at these lowcoordinated sites is suggestive of the hole-hole correlation effects reflected in the line shape of Auger electron spectroscopy. The Auger process creates two holes on an atom, and these two final-state holes once created together cannot separate when the hole-hole repulsion, $U$, is greater than the bandwidth, $\Gamma{ }^{31}$ When the opposite is true, correlation effects can be ignored. The results here suggest the same phenomena, since $U$ may be greater than $\Gamma$ at the low-coordinated corner and face sites in small metal particles; i.e., where the bandwidth is small and the hindrance of screening makes $U$ large. This keeps the maximum hole occupation number on each site to 1 (to push more on would require too much energy). These correlation effects also open up a gap in the DOS, as illustrated in Figure 8 , since to introduce a second hole on a site, an additional energy $U$ must be expended. This gap is called a "correlation" gap in the Mott-Hubbard theory of correlated conduction in solids, where the two bands are called the upper and lower Hubbard bands (UH and LH bands as labeled in Figure 8) and the gap occurs when $\Gamma / U \approx 1.16 .^{24,25,38}$

In Figure 9, at the points where $h(N)$ nears $f(N)$ and the hole occupation number equals 1 , few or no electrons exist above the gap, the UH band is nearly empty, and ICT screening is shut off. This is because ICT screening requires one electron to hop from an adjacent atom to the XAS excited atom, and this will be too costly if the electron has to jump the Hubbard gap to perform this role. The result is little ICT screening under these conditions. This explains the somewhat surprising absence of ICT screening for clusters in the range $N=7.5-9.5$ on supports with electron rich oxygens, just where the UH band is nearly empty, and a gap exists at the Fermi level. Note that in this region the number of interior atoms and holes is quite small, and not until $n_{\mathrm{i}}(N)>0.25$ does the insulator/metal transition occur. Further ICT screening is most easily accomplished when sufficient electrons exist in the UH band, hence when $h(N) \ll$ $f(N)$. This explains the earlier onset of ICT screening on ionic supports as shown in Figure 7, since $h_{\mathrm{c}}$ decreases faster with $N$ on these supports.

Figure 9 now also straightforwardly explains the different onsets for $L_{2}$ band hole occupancy on supports with electron rich vs poor oxygens as found in Figure 4. On covalent supports the $\mathrm{L}_{2}$ band opens at $N=6$, which according to Figure 9 is just below the point where the corner sites reach maximum hole occupancy at $N=6.5$. In contrast, on ionic supports, the $\mathrm{L}_{2}$ band opens at $N=7.5$, just below the point where the face sites reach maximum hole occupancy around 7.9. Obviously, the $\mathrm{L}_{2}$ band will open before the maximum hole occupancy is reached, so that the $\mathrm{L}_{2}$ and $\mathrm{L}_{3}$ bands share the holes as the hole occupancy increases at the respective sites.

C. Comparison with Previously Reported Results. The idea of a reduced valence bandwidth and increased $U$ in lowdimensional metal clusters is of course not new. Theoretical calculations on one-dimensional chains and two-dimensional planes of metal atoms have been reported previously on $\mathrm{Li}, \mathrm{Na}$, $\mathrm{Cu}, \mathrm{Ag}$, and $\mathrm{Au}$ clusters. ${ }^{36,39-42}$ Depending on the type of calculation (unrestricted Hartree-Fock, GVB, or Mott-Hubbard), sp valence band metals exhibit antiferromagnetic spindensity waves and weak delocalization between the interstitial bond orbitals. The insulator phase corresponds to an atomcentered solution (at large atom-atom separation), and the more conducting phase to IBO solutions (at shorter atom-atom distances). Controversy exists whether a Peierls distortion occurs, which helps to open up a gap and bring on insulating character. Calculations on small 1-D and 2-D clusters have also been performed in the framework of the Hubbard Hamiltonian, which takes into account directly the $U / \Gamma$ and band filling. ${ }^{43,44}$ The general conclusions are that a full treatment of electron correlation, i.e., the $U$, is crucial if one is to properly describe 
TABLE 1: Summary of Reported Results on Insulator/Metal Transition in Small Clusters

\begin{tabular}{|c|c|c|c|c|c|}
\hline system & technique & comments & $d(\AA)$ & atoms & ref \\
\hline & & Theory & & & \\
\hline $\mathrm{Fe}, \mathrm{Co}, \mathrm{Ni}, \mathrm{Pd}$ & theory, TB Friedel model & Kubo criterion and bulk metal parameters & $9-11$ & $35-50$ & 45 \\
\hline transition metals Pt, V, Pd & empirical parameters & Kubo criterion and bulk metal parameters & $8-12$ & & 3 \\
\hline $\mathrm{Ni}$ & SCF-TBA & Kubo criterion & & $10-12$ & 46 \\
\hline transition metals & theory & Kubo criterion and bulk $E_{\mathrm{f}}$ as parameter & 5 & 40 & 47 \\
\hline \multicolumn{6}{|c|}{ Photoemission Relative Energy Shifts } \\
\hline $\mathrm{Pd}$ or $\mathrm{Pt} /\left(\mathrm{C}, \mathrm{Al}_{2} \mathrm{O}_{3}, \mathrm{SiO}_{2}, \mathrm{Y}\right.$ zeolite, $)$ & XPS core-level shifts & summary of literature prior to 1989 & $7-12$ & & $\begin{array}{l}48 \text { and refs } \\
\text { therein }\end{array}$ \\
\hline $\mathrm{Pt} / \mathrm{aC}, \mathrm{Pd} / \mathrm{aC}$ & XPS & core-level shift & & 30 & 7,49 \\
\hline $\mathrm{Pd} / \mathrm{aC}$ & XPS & $\mathrm{Pd} 3 \mathrm{~d}_{5 / 2}$ shift & $<15$ & $<50$ & 50 \\
\hline $\mathrm{Pt}$ or $\mathrm{Rh} / \mathrm{Al}_{2} \mathrm{O}_{3} / \mathrm{NiAl}(110)$ & XPS core-level shift & $\Delta E \propto d^{-1}$, no deviation up to $100 \AA$ & $?$ & $?$ & 51 \\
\hline $\mathrm{Pt} / \mathrm{SiO}_{2}$ & PES & energy and/or widths & & $\gg 6$ & 6 \\
\hline $\mathrm{CO} / \mathrm{Pd} / \mathrm{Al}_{2} \mathrm{O}_{3} / \mathrm{NiAl}$ (low $\mathrm{CO}$ cov) & $\mathrm{C} 1 \mathrm{~s}$ XPS and $\mathrm{C} 1 \mathrm{~s}-2 \pi \mathrm{XAS}$ & $\begin{array}{l}\text { "surprising amount of screening already } \\
\text { at } 10 \text { atoms" }\end{array}$ & & 10 & 52 \\
\hline \multicolumn{6}{|c|}{ Bandwidths, STS, and Other Techniques } \\
\hline $\mathrm{Pd} / \mathrm{aC}$ & UPS & Pd VB width & 20 & 140 & 53 \\
\hline Pt carbonyls & $\begin{array}{l}\text { spectroscopic measurement } \\
\text { of gap }\end{array}$ & $\begin{array}{l}\text { gap determined by lowest electronic } \\
\text { excitation }\end{array}$ & & $70-100$ & 54 \\
\hline $\mathrm{Au} / \mathrm{aC}$ & XPS and UPS & $\mathrm{Au} 5 \mathrm{~d}$ band intensity and splitting & $>20$ & $>130$ & 55 \\
\hline $\mathrm{CO} / \mathrm{Pd} / \mathrm{Al}_{2} \mathrm{O}_{3} / \mathrm{NiAl}$ (low $\mathrm{CO}$ cov) & Pd 3d XPS & bandwidths & 20 & 100 & 52 \\
\hline $\mathrm{CO} / \mathrm{Pd} / \mathrm{Al}_{2} \mathrm{O}_{3} / \mathrm{NiAl}$ (high $\mathrm{CO} \mathrm{cov}$ ) & $\mathrm{C} 1 \mathrm{~s}$ XPS and $\mathrm{C} 1 \mathrm{~s}-2 \pi \mathrm{XAS}$ & when C1s IP does not track with XAS edge & $20-25$ & $75-100$ & 52 \\
\hline $\mathrm{Pd} / \mathrm{Al}_{2} \mathrm{O}_{3} / \mathrm{NiAl}$ & cathodluminescence & dramatic increase at onset & $17-22$ & & 56 \\
\hline $\mathrm{Cu} / \mathrm{Al}_{2} \mathrm{O}_{3} / \mathrm{NiAl}(110)$ & $\begin{array}{l}\text { STM height/width } \\
\text { measurements }\end{array}$ & $\begin{array}{l}\text { apparent surface energy change reflects } \\
\text { conductivity change }\end{array}$ & $20-30$ & & 57 \\
\hline $\mathrm{Au} / \mathrm{TiO}_{2}$ & STM $I-V$ curves & 1 and 2 layer clusters & $20-25$ & & 58 \\
\hline $\mathrm{Pd} / \mathrm{TiO}_{2}$ & STM $I-V$ curves & direct gap measurement & & 400 & 59 \\
\hline $\mathrm{Pt} / \mathrm{aC}$ & reactivity & specific activity for $\mathrm{O}_{2}$ reduction & $20-30$ & & 60 \\
\hline
\end{tabular}

the electronic structure and magnetic behavior, and many of these calculations only approximate it.

The nature of the insulator/metal transition in the cited work above involves electron correlation in a narrow $\mathrm{sp}$ band. However considerable work has also appeared on the metal/ insulator transition in materials with narrow $\mathrm{d}$ bands, for example, the transition metal oxides, in particular $\mathrm{NiO} .{ }^{38}$ In the cited work above, the sp or IBO bands were critical. In $\mathrm{NiO}$ the IBO and IBO* bands involving $\mathrm{O}$ are assumed to be separated in energy and contribute insignificantly to the DOS at the Fermi level (we assume the same occurs in the Pt clusters in this work consistent with GVB calculations on small clusters). ${ }^{40}$ In the cited work above, the sp band was assumed to be half full; here the $\mathrm{d}$ band is nearly full, but in both cases around 1 hole per atom exists in a narrow band. In $\mathrm{NiO}$, one generally starts with an atomic Hubbard like Hamiltonian and treats the multiplets of all 10 electrons in the $\mathrm{d}$ band; the multipets then broaden because of the long-range interactions, which may or may not eliminate the gap near the Fermi level. ${ }^{38}$ The theory is complex and not one we wish to discuss here, but the electronic structure in such oxide systems is somewhat similar to that existing at the surface of our Pt clusters. The work reported here is believed to be the first to point out the critical role of the support in rearranging the holes at the surface in small 3-D clusters in such a way as to enable the MottHubbard behavior described here.

Considerable experimental and theoretical work has also been previously reported, where actual predictions of the cluster size at the transition have been made. Table 1 summarizes this work, listing previous estimates of the cluster size where the transition is determined or calculated to occur. Either the cluster diameter or the number of atoms in the cluster (sometimes both) at the point of the transition is often given. At first glance, the data appear to disagree dramatically, with estimates varying from 5 to $30 \AA$ or from 10 to 400 atoms. However, these estimates have been organized by the techniques used to obtain them in Table 1, and some general trends clearly emerge. Table 1 organizes the results into theoretical estimates, those obtained by energy shifts in photoemission, and estimates from other techniques.

(a) Theoretical Kubo Models. In general, the theoretical models assume the Kubo criterion and obtain the DOS from bulk metal parameters. The theoretical estimates for a transition metal such as Pt generally fall around $10 \AA$ or about $35-40$ atoms, although some estimates fall outside this range. These estimates are of course for an unsupported cluster and assume that the DOS are uniform on each atom. Thus it should come as no surprise that the transition point is predicted rather nicely for Pt clusters on acidic supports, since as indicated above, acidic supports counteract the normal distribution of holes in unsupported clusters and potentially produce the most uniform hole distribution. Thus clusters on acidic supports are the most "ideal" with regard to the hole distribution and the point of the insulator/ metal transition.

(b) Photoemission Energy Shifts. The shifts in energy of various photoemission peaks have been the most problematical; estimates for the cluster size at the transition vary over a large range, from 10 to 40 atoms, the smallest of the various estimates. Some reports suggest no transition can be seen at all. This is believed to arise in part because of the problem discussed in the Introduction; XPS leaves a positive charge on the cluster which requires a separation of the contributions arising from screening by the cluster itself and that arising from polarization by the support. 6,7 XAS does not have such a problem, because both the final and initial states are neutral. Nevertheless, taken together the estimates in Table 1 suggest a considerable energy shift occurs (and hence an apparent transition) around 10-35 atoms, or around 7-12 $\AA$, smaller than the theoretical estimates above.

These estimates fall in the range of the turn on of ICT screening, on the ionic and covalent supports, when indeed $\Delta U$ is decreased, and where the photoemission energy shifts should occur. In general photoemission cannot differentiate between ICT and metallic screening, although Sandell et al. ${ }^{52}$ do indeed 
differentiate these effects using the $\mathrm{C} 1 \mathrm{~s}$ XPS and XAS relative energies together for $\mathrm{CO} / \mathrm{Pt} / \mathrm{Al}_{2} \mathrm{O}_{3} / \mathrm{NiAl}$. They indicate that at 10 atoms a "surprising amount of screening already occurs", but they do not attribute this to metallic screening but suggest ICT screening, with the metallic screening coming at much larger cluster sizes. Their estimate of around 10 atoms when the ICT reaches a maximum is in between our results for turn on of ICT screening on ionic and covalent supports, respectively, in Figure 7, but closer to that for the ionic supports. Although $\mathrm{Al}_{2} \mathrm{O}_{3}$ is normally regarded as a relatively intermediate support with respect to covalent character or acidity, the samples of Sandell et al. are prepared by depositing very thin layers $(5 \AA)$ of $\mathrm{Al}_{2} \mathrm{O}_{3}$ on $\mathrm{NiAl}$, and they acknowledge that the metallic alloy $\mathrm{NiAl}$ can be contributing electron density to the $\mathrm{Al}_{2} \mathrm{O}_{3}$. Thus this $\mathrm{Al}_{2} \mathrm{O}_{3} / \mathrm{NiAl}$ support is probably behaving more like an ionic electron rich $\mathrm{O}$ support than a electron poor support.

The estimates for the cluster size at the transition from photoemission energy shifts, although somewhat problematical, can therefore be attributed to the onset of ICT screening, not metallic, and therefore are consistent with Figure 7.

(c) Bandwidths, STS, and Other Techniques. The remaining literature summarized in Table 1 uses a wide variety of techniques to estimate the cluster size at the transition, such as photoemission bandwidths, scanning tunneling spectroscopy, cathodluminescence, and catalytic reactivity. In general these techniques give an estimate for the transition in the range 17$30 \AA$ or around $100-400$ atoms, with the estimates averaging around $25 \AA$. This is exactly in the range where the clusters on ionic supports go metallic according to Figure 7.

Thus these previously reported estimates agree with the data in this work for ionic supports. Most of these data were taken for $\mathrm{Pt}$ or $\mathrm{Pd}$ on $\mathrm{Al}_{2} \mathrm{O}_{3} / \mathrm{NiAl}, \mathrm{TiO}_{2}$, or amorphous $\mathrm{C}(\mathrm{aC}) . \mathrm{TiO}_{2}$ is certainly an ionic support because of its ionic $\mathrm{Ti}-\mathrm{O}$ bond character and the model for the metal support interaction described above. The $\mathrm{Al}_{2} \mathrm{O}_{3} / \mathrm{NiAl}$ was found to behave more like an ionic support for ICT screening as discussed above, so we believe this is indeed behaving as a ionic support. Amorphous carbon $(\mathrm{aC})$ is known to be conductive, so it is not unreasonable to suggest that this will also behave as an electron rich support. Certainly the $\mathrm{aC}$ will not polarize the cluster as described above for an electron deficient oxide support, and the metallic aC can have the same effect on the metal cluster as the apparently more electron rich $\mathrm{Al}_{2} \mathrm{O}_{3} / \mathrm{NiAl}$ support.

A few comments concerning the techniques utilized above are appropriate here. It is well-known that the photoemission energy shifts described above suggest that the transition occurs at smaller clusters while the bandwidth is still much narrower than that for the full bulk metal. Thus several authors have suggested that the bandwidth is a better measure for the onset of the metallic character. In light of the discussion above, this appears to be correct.

Finally, Valden and Goodman, ${ }^{58}$ using scanning tunneling microscopy (STM) current-voltage curves from size-selected $\mathrm{Au}$ particles supported on a single crystal of $\mathrm{TiO}_{2}(110)$ (an ionic/ basic support), observed a dramatic increase in the band gap with decreasing cluster diameter beginning around $20 \AA$ (i.e., suggesting the onset of insulating character). The catalytic activity of these $\mathrm{Au}$ particles toward $\mathrm{CO}$ oxidation underwent a dramatic increase in this diameter range, although appearing to begin and peak about $15-20 \AA$ to larger diameter. Further, the band gap did not appear to be correlated with the thickness of the Au clusters (i.e., 2 layer clusters had a band gap similar to the 1 layer clusters of the same diameter). The band gap appeared to be largest at the surface with some evidence that the interior of the cluster or deeper layers did not have a band gap. This is precisely what is expected based on the model described above, where around $25 \AA$ on ionic supports the metal to insulator transition occurs.

We conclude that the previously reported data, with a few exceptions, are consistent with the results reported here, and this agreement allows us to understand the insulator/metal transition in supported metal clusters.

\section{Conclusions}

In summary, this paper reports the development of a novel probe for assessing the metallic character of supported nanoscale metal clusters and the changing mechanisms for core-hole screening. The ability to perform in situ studies utilizing XAS simultaneously with a determination of the cluster size from EXAFS makes this a powerful technique. The neutral XAS final state, as utilized here, enables a determination of the metallic character expected in the ground state and hence a direct determination of the effects of the support. The technique allows for clear differentiation between local interatomic charge transfer and more diffuse metallic screening.

The results show the dramatic effects the support can have on the metallic and electronic characteristics of metallic clusters. Previously reported estimates of the cluster size at the insulator/ metal transition are organized and correlated and found to be in general agreement with the results reported here. Finally, the importance of the hole distribution detected by this technique and the suggestion of a Mott-Hubbard gap at the surface allow for a much fuller understanding of the insulator/metal transition in supported nanoparticles and clusters.

\section{Appendix}

Although the FEFF8 calculated results above show the proper trends in $R$ at the different sites, the magnitudes in $R$ are not predicted accurately. Figure 5 shows that the unoccupied DOS changes by about $10-50 \%$ upon introduction of the core-hole. Figure 6 shows that the intensity of the calculated $\Delta \mathrm{VB}$ changes by around $30-45 \%$ upon introduction of the core-hole, reflecting this change in final state hole count, $h_{5 / 2}(N)-h_{3 / 2}(N)$. However, the experimental $R(N)$ ratio in Figure 7 changes by only about $15-25 \%$, a factor of 2 smaller. We give two possible reasons:

First, the theoretical calculations reveal that the amount of ICT screening and, hence, $\Delta U(N)$ vary dramatically at the different sites even on the same cluster. Of course the experiment effectively averages over these different sites in a cluster, and the average change will be smaller than at a specific site where ICT screening is large.

Second, the large reduction in the experimental $R(N)$ range is more likely a direct reflection of the dynamic screening mechanisms. Many of the dynamic screening effects mentioned above can be approximated by utilization of the "orthogonalized" final state rule, ${ }^{13-15,25-28}$ which states that the spectral line shape is best approximated by the final state (i.e., in XAS the metallic or ICT-screened core-hole state), but the intensity is determined by the projection of this final state onto the initial no-core-hole state. The remaining intensity appears as shakeoff or shake-up satellites, which usually blend into the background in XAS. This projection will decrease the magnitude of the intensity variations in $\triangle \mathrm{VB}$, but this effect is not included in the FEFF8 calculations.

Thus, FEFF8 overestimates the magnitude of the variations in $R(N)$ with $N$ but correctly predicts the trends. 


\section{References and Notes}

(1) Haberland, H. Clusters of Atoms and Molecules. I: Theory, Experiment and Clusters of Atoms; Springer Series in Chemical Physics, Vol. 52; Springer-Verlag: Berlin, 1995.

(2) Edwards, P. P.; Johnston, R. L.; Rao, C. N. R.; Tunstall, D. P.; Hensel, F. Philos. Trans. R. Soc. London 1998, 5, A356.

(3) Halperin, W. P. Rev. Mod. Phys. 1986, 58, 533.

(4) Dowben, P. A. Philos. Trans. R. Soc. London 1998, 267, A356.

(5) Hunt, M. R. C.; Palmer, R. E. Philos. Trans. R. Soc. London 1998 A356, 231.

(6) Eberhardt, W.; Fayet, P.; Cox, D. M.; Fu, Z.; Kaldor, A.; Sherwood, R.; Sondericker, D. Phys. Rev. Lett. 1990, 64, 780.

(7) Wertheim, G. K.; Di Cenzo, S. B. Phys. Rev. 1988, B37, 844.

(8) Mojet, B. L.; Miller, J. T.; Ramaker, D. E.; Koningsberger, D. C. J. Catal. 1999, 186, 373-386.

(9) Vayssilov, G. N.; Rösch, N. J. Catal. 1999, 186, 423-432.

(10) Koningsberger, D. C.; Oudenhuijzen, M. K.; Ramaker, D. E.; Miller,

J. T. Stud. Surf. Sci. Catal. 2000, 130A, 317-322.

(11) Vaarkamp, M.; Mojet, B. L.; Modica, F. S.; Miller, J. T.; Koningsberger, D. C. J. Phys. Chem. 1995, 99, 16067.

(12) Mattheiss, L. F.; Dietz, R. E. Phys. Rev. B 1980, 22, 1663-1676.

(13) von Barth, U.; Grossmann, G. Phys. Rev. B 1982, 25, 5150; Phys.

Scr. 1980, 21, 580; Solid State Commun. 1979, 32, 645.

(14) Anderson, P. W. Phys. Rev. 1961, 124, 41.

(15) Economou, E. N. Green's Functions in Quantum Physics; Springer Series in Solid-State Sciences, Vol. 7; Springer-Verlag: Berlin, 1979.

(16) Mahan, G. D. Phys. Rev. B 1980, 21, 1421.

(17) Gadzuk, J. W. J. Electron Spectrosc. Relat. Phenom. 1977, 11, 255.

(18) Davis, L. C.; Feldkamp, L. A. Phys. Rev. B 1981, 23, 4269.

(19) Grebennikov, X. X.; Babanov, Y. A.; Sokolov, O. B. Phys. Status Solidi 1977, 80, 73.

(20) Gunnarsson, O.; Schonhammer, K. Phys. Rev. B 1980, 22, 3710 Schonhammer, K.; Gunnarsson, O. Solid State Commun. 1977, 23, 691.

(21) Fröhlich, H. Physica 1937, 4, 406.

(22) Kubo, R. J. Phys. Soc. Jpn. 1962, 17, 975.

(23) Alonso, J. A. Chem. Rev. 2000, 100, 637-677.

(24) Mott, N. F. Proc. Phys. Soc. (London) 1949, A62, 416; Philos. Mag. 1961, 6, 287. Mott, N. F. Metal-Insulator Transitions; Barnes and Noble: New York, 1974.

(25) Hubbard, J. Proc. R. Soc. London, Ser. A 1964, 276, 238; 1963 , 277, 237; 1964, 281, 401.

(26) McAdon, M. H.; Goddard, W. A., III Phys. Rev. Lett. 1985, 55, 2563; J. Phys. Chem. 1987, 57, 2607. Kua, J.; Goddard, W. A., III J. Phys. Chem. B 1998, 102, 9481; 1998, 102, 9492.

(27) Ankudinov, A. L.; Ravel, B.; Rehr, J. J.; Conradson, S. D. Phys. Rev. B 1998, 58, 7565-7576.

(28) de Graaf, J. Ph.D. Dissertation, Utrecht University, Ridderkerk, The Netherlands, 2001.

(29) de Graaf, J.; van Dillen, A. J.; de Jong, K. P.; Koningsberger, D. C. J. Catal. 2001, 203, 307-321.

(30) Wolff, P. A. Phys. Rev. 1961, 124, 1030.
(31) Ramaker, D. E. Solid State Mater. Sci. 1991, 17, 187.

(32) López, N. J. Chem. Phys. 2001, 114, 2355-2361.

(33) Feibelman, P. J.; Hamann, D. R. Surf. Sci. 1985, 149, 48.

(34) Ramaker, D. E.; Mojet, B. L.; Koningsberger, D. C.; O’Grady, W. E. J. Phys. Condens. Matter 1998, 10, 8753-8770.

(35) Tong, Y. Y.; Mérlaudau, P.; Renouprez, A. J.; van der Klink, J. J. J. Phys. Chem. B 1997, 101, 10155.

(36) Balbuena, P. B.; Derosa, P. A.; Seminario, J. M. J. Phys. Chem. B 1999, $103,2830$.

(37) Gordon, M. B.; Cyrot-Lackmann, F.; Desjonqueres, M. C. Surf. Sci. 1977, 68, 359. Benfield, R. E. J. Chem. Soc., Faraday Trans. 1992, $88,1107$.

(38) Adler, D.; Feinleib, J. Phys. Rev. B 1970, 2, 3112.

(39) Lepetit, M. B.; Apra, E.; Malrieu, J. P.; Dovesi, R. Phys. Rev. B 1992, 46, 12974.

(40) McAdon, M. H.; Goddard, W. A., III J. Phys. Chem. 1988, 92, 1352.

(41) Baraille, I.; Lepetit, M.-B. J. Phys. Chem. 1996, 100, 4224

(42) Tatewaki, H.; Tomonari, H. M. Can. J. Chem. 1992, 70, 642.

(43) Lopez-Urias, F.; Pastor, G. M. Phys. Rev. B 1999, 59, 5223.

(44) Ojeda, M. A.; Dorantes-Davila, J.; Pastor, G. M. Phys. Rev. B 1999, 60,6121 .

(45) Zhao, J.; Chen, Z.; Wang, G. Phys. Rev. B 1994, 50, 15424.

(46) Aguilera-Granja, F.; Bouarab, S.; Vega, A.; Alonso, J. A.; Montejano-Carrizales, J. M. Solid State Commun. 1997, 104, 635.

(47) Harrison, M. R.; Edwards, P. P. The Metallic and Nonmetallic States of Matter; Edwards, P. P., Rao, C. N. R., Eds.; Taylor and Francis: London, 1997; pp 389-416.

(48) Che, M.; Bennett, C. O. Adv. Catal. 1989, 36, 55.

(49) Wertheim, G. K.; DiCenzo, S. B.; Buchanan, D. N. E. Phys. Rev $B$ 1986, 33, 5384 .

(50) Kuhrt, Ch.; Harsdorff, M. Surf. Sci. 1991, 245, 173.

(51) Freund, H. J.; Bäumer, M.; Kuhlenbeck, H. Adv. Catal. 2000, 45, 333.

(52) Sandell, A.; Libuda, J.; Brühwiler, P. A.; Andersson, S.; Bäumer, M.; Maxwell, A. J.; Mårtensson, N.; Freund, H.-J. Phys. Rev. B 1997, 55 , 7233; J. Electron Spectrosc. Relat. Phenom. 1995, 76, 301; J. Vac. Sci. Technol. A 1996, 14, 1546.

(53) Unwin, R.; Bradshaw, A. M. Chem. Phys. Lett. 1978, 58, 58

(54) de Biani, F. F.; Femoni, C.; Iapalucci, M.; Longoni, G.; Zanello,

P.; Ceriotti, A. Inorg. Chem. 1999, 38, 3721.

(55) Lee, S. T.; Apai, G.; Mason, M. G.; Benbow, R.; Hurych, Z. Phys. Rev. B 1981, 23, 505 .

(56) Adelt, M.; Nepijko, S.; Drachsel, W.; Freund, H.-J. Chem. Phys. Lett. 1998, 291, 425.

(57) Worren, T.; Hansen, K. H.; Laegsgaard, E.; Besenbacher, F.; Stensgaard, I. Surf. Sci. 2001, 477, 8.

(58) Valden, M.; Goodman, D. W. Science 1998, 281, 1647.

(59) Xu, C.; Lai, X.; Zajac, G. W.; Goodman, D. W. Phys. Rev. B 1997, $56,13464$.

(60) Kinoshita, K. J. Electrochem. Soc. 1990, 137, 845 